\title{
Origin of Order: Emergence and Evolution of Biological Organization as a Problem in Thermal Physics
}

\author{
Fouad El-Diasty
}

Physics Department, Faculty of Science, Ain Shams University Abbasia Cairo 11566 Egypt

\begin{abstract}
According to a mechanistic and naturalistic view of the origin of life, the reality is evolution - a single process of self-transformation started from a primordial chaotic or random state of matter reaching to modifications through natural selection. The simplest form of life requires thousands of different kinds of molecules, to produce very complex protein DNA and RNA molecules subunits arranged in a precise sequence. Chemical and physical processes acted for many hundreds of millions of years, before true cellular life was brought into being. Biological evolution was able to create complex, harmonic, and very effective biocybernetic control systems governing the DNA behavior and the evolution of animal cognition abilities. But the present postulates on the origin of life do not satisfy the criteria of a scientific immensity theory, where all related laboratory experiments are mere exercises in organic chemistry. The immensity of the problem is appreciated by physics through applying the thermodynamics laws which are indeed universal. The catalysts in biological systems are coded in the genes of the DNA, so is a fourth law needed for thermodynamics about the self-organizing phenomena observed in biology? Can the physics-based theories robustly address phenomena of emergence and evolution without having recourse to the type of vitalism that was in the beginning of the twentieth century? Is the origin of life beclouded because we don't know enough about thermodynamics? Why this should be called a scientific attitude when all the scientific evidences continue to support special creation, or indeed an omnipotent Creator?
\end{abstract}

Keywords Second Law Of Thermodynamics, Entropy, Law Of Temporal Hierarchies, Supramolecular Thermodynamics, Biological Evolution

"Say, roam the earth and see how the creation was started....."

The Qura'an, surat Al Ankabut 29 (the spider) verse 20

\section{Introduction}

The origin or emergence of life is one of the most important questions facing scientists. Why a specific material system is an organism and not something else or how life might have arisen. Biological evolution as genetic changes in populations of organisms over successive generations is an irreversible process of the historical variation of life. Charles Darwin and Alfred Wallace's theories and the modern synthetic theory of evolution emphasize the evolution of populations. These theories study the causes, mechanisms and general rules of the evolution of living organisms from the biological point of view. The development of modern Darwinism is connected with the analysis of the data obtained by molecular biology. Life on earth was physically initiated in accordance with the second law of thermodynamics (entropy) and the law of temporal hierarchies. The

* Corresponding author:

fdiasty@yahoo.com (Fouad El-Diasty)

Published online at http://journal.sapub.org/als

Copyright (C) 2011 Scientific \& Academic Publishing. All Rights Reserved cell is an open system with thermodynamic "dissipative structure' reducing it to physics of "organized complexity". Dissipative structures in physical and chemical systems are phenomena that are explained by nonequilibrium thermodynamics, kinetic theory and the law of temporal hierarchies. However, building a coherent and internally consistent picture that allows the formulation of a hypothesis which places living (animate) and inanimate matter within a single conceptual framework is still missing. The central aim of this article is to review the governing physical principles of living and non-living systems and to clarify the nature of these principles on evolution transformation and human conscience.

\section{Physics as Thermodynamics}

Physics is the science which concerns with the relations that governing the four physical building blocks of our material world (universe). These four global aspects are matter, energy, space and time regardless of what is the philosophy behind. Four types of energy are known (electromagnetic, gravitational, strong and weak nuclear energies) the most important type of energy for topic is the electromagnetic one. This kind of energy has different apparent forms and effects in our common sense; for example heat, light, elec- 
tricity and mechanical motion. In addition it is responsible for the existence of the periodic elements and their chemical reactions.

On the other hand, thermodynamics is the field of physics that deals with the direction of flow, transformation, storage, dissipation of thermal energy (heat) and the interrelation between heat and other forms of energy. Thermodynamics is also concerned with systems of very large numbers of particles so that thermodynamic variables, such as pressure, volume, and temperature, are considered as statistical quantities. This field of science is concerned with the changes of energy and not with the mechanisms by which such changes occur.

\subsection{Second Law of Thermodynamics "Entropy"}

The conservation law, or the first law of thermodynamics, states that energy can neither be created nor destroyed. This provides the basis for all quantitative accounts of energy, regardless of its form. Therefore, in a closed system, the total amount of energy of all kinds is constant and the heat absorbed by the system is equal to summation of the increase in the internal energy plus the work done by the system. The studies of William Thomson (Lord Kelvin) (1851), Carnot (1824), and Clausius (1850) of the exchanges of energy in thermal machines revealed that there is a hierarchy among the various forms of energy and an imbalance in their transformations. These hierarchy and imbalance are the basis of the formulation of the second law. The irreversible increase of the nondisposable energy in the universe is measured by an abstracted dimension that Clausius in 1865 called entropy (from the Greek entrope, change).

The entropy law, or the second law of thermodynamics, deals with the direction in which any chemical or physical process involving energy takes place, where no self-acting mechanism can transfer heat from a colder to a hotter body and produce no other external effect. Thus this law states that in all processes some of the energy involved irreversibly (spontaneously) loses its ability to do work and is degraded in quality. The latter is called thermodynamic entropy whose extreme form is dispersed heat and manifested in a uniform temperature distribution.

Another statement of this second law is that in any closed system the process entropy never decreases. The idea was framed more dramatically by Helmholtz in 1854 providing speculate of the heat death of the universe. The irreversibility of physical processes makes the entropy law probably the most important law for understanding physical processes and hierarchical systems including living organisms and even social relations and situations.

The asymptotic law, or the third law of thermodynamics, was first formulated by Walther Nernst (1906). Max Planck (1913) developed the law depending on a statistical point of view. The law confirms that if a chemical change occurs between pure crystalline solids at absolute zero, there is no change in entropy, i.e. the entropy of the final substance equals that of the initial substances. Accordingly, all processes slow down as they operate nearer to the thermodynamic equilibrium (irreversible processes). It makes reaching the thermodynamic equilibrium difficult in practice. The law suggests that the fast powerful and powerful changes which are characteristics of technology and living forms occur only at levels far distant from thermodynamic equilibrium.

Equilibrium-based thermodynamics (spontaneous processes) does not use time as a parameter and does not consider the mechanism of the phenomenon. Thermodynamics answers the question: where is the process directed before equilibrium can be achieved? The work of Rumford, Joule, Mayer, Clausius, Carnot, Kelvin, and others established the concept of heat as a form of energy. The kinetic theory through the work of Gibbs, Maxwell, and Boltzmann combines this conclusion with molecular chemistry and interprets the heat content of a system (body) as a molecular motion of which the system is made up. The system is reduced to a state of maximum disorder in which each individual movement is neutralized by statistical laws. Accordingly, potential energy is organized energy while heat is disorganized energy. Indication of molecular agitation is afforded by diffusion and Brownian motion. Therefore, kinetics studies the rates of processes and their mechanisms.

There are at least three ways of defining entropy: (1) thermodynamically (the science of heat), where the names of Kelvin, Mayer, Joule, Carnot, and Clausius (1865) are important, (2) in terms of statistical theory, which fosters the equivalence of entropy and disorder - as a result of the work of Maxwell, Gibbs, and Boltzmann (1875), and (3) in terms of information theory, which demonstrates the equivalence of neguentropy (the opposite of entropy) and information - as a result of the work of Szilard, Gabor, Rothstein, and Brillouin (1940-1950).

The concepts of entropy and irreversibility, derived from the second principle, have changed our view of the nature and universe. In being tackled with biological evolution generating order and organization, the concept of entropy indirectly opens the way to a philosophy of progress and development. The thought that by the nature of entropy the ultimate and only possible future for man is annihilation led Leon Brillouin to ask, "How is it possible to understand life when the entire world is ordered by a law such as the second principle of thermodynamics, which points to death and annihilation?

The second law of thermodynamics states that in an isolated system the entropy will increase. In the statistical definition of entropy according to Boltzmann, this means that the system will evolve to its most probable state, that is, the one with the most homogeneous probability distribution. Consider the classical example: a closed box with two compartments where the left compartment contains gas and the right one is empty. Making a hole in the wall separating the two compartments leads to the gas will be sucked out of the full compartment into the vacuum of the empty one, until both compartments contain equal amounts of gas. 
When the concentration of molecules in the two compartments is uniform will there be an equal flow through the hole in both directions. This configuration where the distribution is homogeneous is the one with maximum entropy.

However, since the amount of gas molecules in either compartment is so large, a very large number of them must move "countercurrent" to produce a noticeable difference. The probability that such a vast number of molecules would all move together in the same direction is vanishingly small; so small that we can assume that it will never take place. Because of the law of large numbers, the larger the number of coincidences needed for an effect to occur, the smaller the probability that the effect will occur. And with numbers as large as the number of molecules in a gas, the resulting probability is as close to zero. Since the movement of the microscopic particles cannot be predicted, the evolution towards homogeneity on the macroscopic scale is perfectly predictable. On the microscopic level of the particle, all directions of change have the same probability. On the macroscopic level of the gas, however, one direction of change-towards greater homogeneity-has a much larger probability. This preferred direction creates a "time direction". Time always flows in the direction of increased entropy, i.e., greater homogeneity. Although, thermodynamics has introduced irreversible change into the scientific world view, it fails to explain the development of new organization which might be associated with evolution.

\subsection{Photosynthesis as Non-Equilibrium Thermodynamic Model}

Much of the recent theorizing about the thermodynamics of evolution attempts to build a model up from non-living systems[1]. The most basic thermodynamics of humans and of other organisms is how to extract as much useful work from a potential energy gradient as possible; providing a minimum value for Gibbs function of formation. There are two ways to do this assumption. One way is to increase the magnitude of energy flow across the gradient. This can lead to increased entropy production, if the thermodynamic efficiency of the process remains constant. The second way is to increase the (thermodynamic) efficiency of its use, i.e. maximize the work extracted and minimize the entropy production. The second approach, which seems to be highly optimized in natural systems, may actually lead to a reduction in the entropy raise. Thus, in many cases living systems actually create less entropy, t2han comparable non-living systems operating across the same potential gradient over a given period of time. The second law cannot be violated but it can be over-involved, where in that sense life could be regarded as energy "kiting" scheme.

Let us examine the thermodynamic behavior of organisms engaged in photosynthesis. The energy from photons coming from the sun is converted to potential energy in the form of excited electrons and used to do work. Thus the solar energy creates higher energy molecules in the form of carbohydrates and molecular oxygen in the atmosphere. The solar energy is captured and used for photosynthesis, where about $20 \%$ of this energy actually striking a leaf and is converted into chemical potential energy[2]. This energy is bound up in the carbon compounds which make up the structure of the organism, i.e. organic material, and in the higher chemical potential energy state of the atmosphere. This portion of the incident solar energy is not immediately reradiated in the form of thermal dispersion, and so it does not immediately contribute to increased entropy. Therefore, throughout the time with photosynthesizing organisms on earth, the overall rate of entropy production on earth has in fact been less than it would otherwise have been without them. The difference is seen as the energy sequestered in the vast accumulation of biomass and fossil fuels on earth.

\subsection{Cultural and Social Entropy}

Social entropy is a measure of the natural decay of the structure or of the disappearance of distinctions within a social organization. Much of the energy consumed by a social organization is spent to maintain its structure, counteracting social entropy, e.g., through legal institutions, education, the normative consequences or television. Anomie is the maximum state of social entropy. Also anomie is a condition of disintegration of a society into individual components resulting from the absence of conventions, shared perceptions and goals. A social system is describable as a simple aggregate, i.e., the state of maximum social entropy. Social system in cybernetics is a system involving its observers. Such a system is constituted by communication among observers who participate within that system by drawing distinctions and creating relations within it. On the other hand negentropy (inverse of entropy) is a measure of the complexity of a physical structure in which quantities of energy are invested, e.g., buildings, technical devices, organisms but also atomic reactor fuel, the infrastructure of a society. In this sense organisms may be said to become more complex by feeding not on energy but on negentropy.

\subsection{The Direction of Evolution}

Functioning of biological systems is possible on the condition of sufficient "permeability" for the matter-building material of the supramolecular structure. Internal and external forces should exist to enhance "mixability" inside the system, e.g., metabolism. Periodic oscillations of the environmental parameters (thermostat) around the mean values play the role of such forces. Hence, the combined effects of internal thermodynamic factors (expressed inside the system) and external thermodynamic effects (changes and oscillations of the environmental physical parameters) determine the direction of evolution[3]. This model reveals special attention to the physical chemistry of the supramolecular structures, which should be considered as a "key" for understanding biological evolution.

Although evolution is chaotic and unpredictable, it moves preferentially in the direction of increasing complexity or fitness which implies a preferred direction for evolution, a 
"progress" towards more sophisticated forms[4]. Recent advances in evolutionary theory (such as the theory of punctuated equilibrium) and observation of evolutionary phenomena seem to indicate that evolution is a largely unpredictable, chaotic and contingent series of events, where small fluctuations may lead to major catastrophes. For example, consider a rock that rolls down from the top of a steep mountain. The slightest irregularity in the terrain may be sufficient to make the exact path of the rock virtually impossible to predict. One thing will be certain: the final position will be lower than the initial position at the top.

Apply this metaphor to evolution, the "vertical" dimension or variable that can only increase during evolution is entropy. Fundamental theorem of natural selection[5] has shown that for populations of living systems the average fitness is the variable. This is due to the fact that fit individuals by definition will become more numerous while the proportion of less fit individuals will decrease. Fitness is a relative notion; what is fit in one type of environment may no longer be fit in another environment. Thus, the inexorable increase of fitness only holds in invariant environments. For example: the evolution from hairless elephant to woolly mammoth is due merely to a cooling down of the climate. If the climate becomes warmer again the woolly variant will lose its fitness relative to the hairless one, and the trend will be reversed.

There are different ways to increase fitness. First, the system may increase its internal or intrinsic fitness by adding or strengthening bonds or linkages between its components. This is typically accompanied by the increase of structural complexity. Second, the system may increase its fitness relative to its environment by increasing the variety of environmental perturbations that it can cope with, and thus its functional complexity. Though the warm-blooded, woolly mammoth is only relatively fitter than its hairless cousin, it is absolutely fitter than a cold-blooded reptile. Warm-bloodedness means temperature control (metasystem transition), i.e. the capacity to internally compensate a variety of fluctuations in outside temperature. A system that can survive situations $\mathrm{A}, \mathrm{B}$ and $\mathrm{C}$, is fitter than a system that can only survive A and B. Thus, evolution will tend to irreversibly produce increases of functional complexity. These systems can be ordered by their functional complexity, the resulting relation is not a linear order but a partial order. In general, there is no absolute way in which one can know whether a system that can survive situations $\mathrm{A}, \mathrm{B}$ and $\mathrm{C}$ is more or less complex or fit than a system that can survive C, $\mathrm{D}$ and E. One can state that both systems are less fit than a system that can survive all A, B, C, D and E.

Mathematically, such a partial order can be defined by the inclusion relation operating on the set of all sets of situations or perturbations that the system can survive. The first mentioned system might add either D or E to the set of situations with infinite number of possibilities. This situation leaves evolution wholly unpredictable and open-ended. For example, humans are in all likeliness absolutely more functionally complex than snails or frogs, evolution might well have produced a species that is very different from humans with a much higher functional complexity level compared to the other species.

\subsection{Origin and Emerging of Life}

In biology textbooks life is defined in terms of distinctive properties that distinguish living systems from non-living. Life for biologists is simply the subject of interest. Debate between the "mechanists" and the "vitalists" about the relationship of matter and life as well as matter and mind is spilled over the twentieth century, the century of biochemistry and molecular biology, artificial intelligence, artificial life, and complex systems theory. Something that is alive entities metabolize, grow, die, reproduce, respond, move, heritable variability, producing new and emergent functional structures that provide increased adaptive fitness in changing environments.

Independently in the 1920 s A. Oparin and J.B.S. Haldane $[6,7]$ proposed the first modern hypotheses which are considered as a metabolism-first view for how life originated on earth. On the primitive earth the geophysical conditions were quite different from the present, where no molecular oxygen in the atmosphere was existed (oxygen arising very much later in time with the appearance of photosynthetic organisms that used light energy to split water). In this chemically reducing atmosphere an increasingly complex "soup" or organic molecules would arise from which the precursors of living systems could arise.

Another sequence to living things were proteins (the polymers of amino acids formed under conditions of high temperature), where some amino acids could be produced by the action of an electrical discharge through a mixture of gases thought to be present in the primitive atmosphere. The protein-first view suggested that the chemistry that lead to life could have occurred in some weak catalytic activity that facilitated the production of the other needed molecular components. The abiotic routes to nucleic acids are considered after understanding the structure of DNA. Dawkins[8] assumed a nucleic acid, formed by chance, would be the start of life since it would "self replicate". The postulate of not only nucleic acids first but an 'RNA world' is raised because RNA is capable of some catalytic activity[9]. Taking into consideration that some type of metabolism would be needed to sustain RNA replication.

Proto-cell-first approach[10-13], congenial to a thermodynamic and systems emphasizes the need to presence of the main factors that distinguish cells from non-cells: metabolism via autocatalytic cycles of catalytic polymers, replication, and a physical enclosure within a chemical barrier like that provided by the cell membrane. Together with the emergence of the first entities or living comes the emergence of biological selection in which contingency plays a much greater part.

Darwin bracketed the question of the origin of life from questions of descent with modification through natural selection. Indeed, Darwinian theories of evolution can take living systems as a given and then explore how novelties 
arise through a combination of chance and necessity. However, an understanding of how life might have emerged would provide a bridge between our view of the properties of living systems and the evolutionary phenomena they exhibit. Such an understanding is needed to anchor living systems in matter and the laws of nature[14]. This remains a challenge to be met in order for science to provide a more full answer to the question what is the origin of life.

Life as a pattern of chemical processes involves not only the replication of the DNA that carries the genetic information but the epigenetic building of the organism through a sequence of developmental steps. Evolutionary phenomena are an inextricable aspect of living systems; any attempt to study life in the absence of this diachronic perspective will be futile. Thus the biological systems may be defined as open systems maintained in steady-states, farfrom-equilibrium, due to matter-energy flows in which informed (genetically) autocatalytic cycles extract energy, build complex internal structures, allowing growth even as they create greater entropy in their environment.

\subsection{Power of Replication}

Replication first school of thought, as opposed to the metabolism first school, is pioneered by Oparin[15] in the beginning of the $20^{\text {th }}$ century. Forty years ago Eigen[16,17] suggested that the life began with the appearance of a replicating molecule. It then evolved by an imperfect replication and chemical selection into early life forms. The life began with a primal and a simple molecular replication reaction, where the replication reaction (autocatalytic) has unique kinetic properties[17,18]. Lifson[18] has provided a numerical example; for instant consider the two reactions[19]:

$$
\begin{aligned}
& A+B \stackrel{X}{\longrightarrow} C \\
& A+B \stackrel{X}{\longrightarrow} X
\end{aligned}
$$

Reaction (1) represents any chemical process where reactants $A$ and $B$ are converted into product $C$ through the catalytic effect of $X$. Reaction (2), the molecular replication reaction, is an autocatalytic reaction, in which the catalyst $X$ converts $A$ and $B$ into more of itself. For reaction (1), assume a single molecule of catalyst $X$ and an arbitrary reaction rate of $10^{-6} \mathrm{~s} /$ molecule, a period of $6 \times 10^{17} \mathrm{~s}$ (derived from $6 \times 10^{23} \times 10^{-6} \mathrm{~s}$ ) or 20 billion years would be required in order to generate a mole of product, $C$. For reaction (2), due to the huge kinetic power of replication, it would take just a tiny fraction of a second for a mole of product, $X$, to be generated!

The mathematics of replication is such that a single replicating molecule undergoing some 79 acts of replication becomes a mole $\left(6 \times 10^{23} \approx 2^{79}\right)$, so it would take just $79 \times 10^{-6}$ $\mathrm{s}$ for a molecule to become a mole. The relative magnitude of these two figures, 20 billion years for catalytic reactivity and $79 \mathrm{~ms}$ for autocatalytic reactivity, though the enormous kinetic potential associated with the replication reaction places it in a unique kinetic category. Thus the autocatalytic replication reaction, by definition, is an extreme expression of kinetic control, and will tend to overwhelm any competing reaction, thermodynamically preferred or not.

\subsection{Biochemical Conception of Life}

According to Hopkins[20], the life is a property of the cell as a whole, because it depends upon the organization of processes. He saw that the cell is a chemical machine obeying the laws of thermodynamics and physical chemistry, but having organized molecular structures and functions. The chemistry underlying metabolism was catalyzed and regulated by enzymes, protein catalysts, and small changes in structure and energy of well-defined chemical intermediates. The living cell is not a mass of matter composed of a congregation of like molecules, but a highly differentiated system. The cell, in the modern phraseology of physical chemistry, is a system of co-existing phases of different constitutions. Understanding how the organization was achieved is just as important as knowing how the chemical reactions occurred.

There was a challenge for Hopkins to figure out how rather simple physical and chemical laws could produce the complexity of living systems. By the time of World War II it was meaningful to address the question of "what is life?" in molecular terms and fundamental physical laws. It was clear that there were several distinct ways in which matter in living systems behaved in ways different from non-living systems. How could genetic information be instantiated at a molecular level given that ensembles of atoms or molecules behaved statistically? How could biological systems generate and maintain their internal order in the face of the imperative of the second law of thermodynamics that all natural systems are processed with increasing entropy?

\subsection{Schrödinger's Dual Legacy and the Meaning of Life}

In 1944 Schrödinger provided, in his book "What is Life?" [21] (after an obscure but groundbreaking article by Lotka [22]) a major impact on the development of twentieth century biology, especially upon Francis Crick and James Watson[23] and other founders of molecular biology. Schrödinger gathered together several strands of research and stated his questions in a stark and provocative manner. Max Delbrueck demonstrated that the size of the mutations caused by X-rays had the dimensions of a molecule of a thousand or so atoms.

The first Schrödinger question was how it could be sustained order in the molecules responsible for heredity when the statistical ensembles of molecules quickly became disordered (with increased entropy as predicted by the second law of thermodynamics). The problem of heredity was then reformulated at the molecular level as to how order could give rise to order? The second question for Schrödinger was concerned the thermodynamics of living things in general, that is, how could they generate order from disorder through their metabolism? It was through answering these two specific questions from the perspective of a physicist that Schrödinger sought to answer the big question, what is life? 
Schrödinger argued that the molecular material had to be an aperiodic solid that had a miniature code in its structure. The pattern of constituent atoms comprising the molecule of heredity would have a higher-level order due to the pattern of its molecular subunits. This higher-level has aperiodic order that would contain the coded information of heredity. The elucidation of the structure of DNA and the explosion of molecular genetics has eclipsed the other, but with respect to Schrödinger the most important aspect of metabolism is that it represents the cell's way of dealing with all the entropy that produces as it builds its internal order "negentropy". He noted that the cell must maintain itself in a state away from equilibrium since thermodynamic equilibrium is the definition of death.

By creating internal order and organization within a living system (cells, organisms or ecosystems) the metabolic activities must produce greater disorder in the environment, such that the second law is not violated. Order from order and order from disorder, tied together by claiming, an organism concentrates a 'stream of order' on itself and thus escaping the decay into atomic chaos of 'drinking orderliness' from a suitable environment. It seems to be connected with the presence of aperiodic solids and the chromosome molecules which doubtless represent the highest degree of well-ordered atomic association - much higher than the ordinary periodic crystal - in virtue of the individual role every atom and every radical is playing. Although Schrödinger was giving a physicist's answer, he did not confine himself only to the question of what distinguished the living from the non-living and in the epilogue reflects on free will and consciousness. The issue of consciousness was seen by Schrödinger too complex as connected to that of life itself.

It is less known how organisms gain order from disorder through the thermodynamics of open systems far from equilibrium (nonequilibrium thermodynamics) and how organisms produced their internal order while affecting their environment not only by their activities but also through created disorder in it. The self-replicating DNA has become a major metaphor for understanding life. The world is increasingly divided into replicators to control development representing fundamental level of action for natural selection.

The emergent, self-organizing spatial-temporal patterns are seen not only in cells and organisms, but in ecosystems. What so important are the dynamics of non-linear interactions (where the responses of a system can be much larger than the stimulus) and autocatalytic cycles (reaction sequences that are closed on themselves where a larger quantity of one or more starting materials is made through the processes). Given that the catalysts in biological systems are coded in the genes of the DNA, thus living systems are seen as informed, autocatalytic cyclic entities that develop and evolve under the dual dictates of the second law of thermodynamics and of natural selection.

Living beings exhibit complex functional organization (inherently involves functions and their interrelations) and the ability to adapt their environments over generational time, representing the challenge to physically-based explanations based upon mechanistic (reductionistic) assumptions. A "fourth law" of thermodynamics about such phenomena may be needed. The physics-based theories can robustly address phenomena of emergence without having recourse to the type of "vitalism" that was in the earlier part of the twentieth century.

\subsection{The Problem of Harmonizing Entropy with Evolution}

According to the Nobel Prize committee, the work of Prigogine is building a bridge between the physical and biological sciences[24,25]. He had shown that the tremendous amount of information necessary for molecular self-replication can be produced naturalistically despite the entropy law. He has offered a theoretical speculation, not an experimental demonstration. According to a principle as "order through fluctuations" the biological structures are created by the continuous flow of energy and matter from the outside world. Their maintenance requires a critical distance from equilibrium, that is, a minimum level of dissipation with a higher degree of structure, or order.

Also their generation is accompanied by a large dissipation of energy in the form of heat. With the formation of convection currents and vortices in a fluid subjected to a temperature gradient, vortices (or other fluctuations or instabilities) may be generated and maintained. This phenomenon is known in hydrodynamics but Prigogine suggested that it can be applied in certain chemical and biological reactions which are proceeding under non-equilibrium conditions. Dissipative structures require the dissipation of much flow energy in the form of heat for their generation.

As far as chemical or biological reactions are concerned, the generation of dissipative structures is limited to auto-catalytic processes. But catalytic processes, like fluid vortices, do not generate higher order going downhill thermodynamically. The auto-catalytic processes would require already-living systems for their own generation, so they can hardly explain the generation of living systems! Prigogine's analysis failed to solve the problem of harmonizing entropy with evolution and he has not shown that life can evolve from non-living chemicals. His dissipative structures do not constitute either the required program or the required mechanism to enable any kind of permanently increased order to be produced in an open system.

The problem of the origin of life can really only be resolved by recognition of the omnipotent Creator. The only alternative to belief in special creation is credulous faith in impotent chance. Genesis was a statistically unlikely event, but certainly it occurred. Was there a temporary repeal of the second law that permitted a "fortuitous concourse of atoms"? It may hold with the more traditional scientific attitude that the origin of life is beclouded merely because no one knows enough about thermodynamics and other conditions on the earth many eons ago. Why this should be called the scientific attitude when all the scientific evi- 
dences continue to support special creation.

\section{Hierarchical Thermodynamic Theory}

The study of thermodynamic systems far from equilibrium (nonequilibrium thermodynamics), or synergetics, is far applicable only to certain phenomena based on pure kinetic methods. It led to that the evolution of living systems cannot agree with the second principle of thermodynamics[26]. To overcome this problem, a new hierarchic thermodynamics or macrothermodynamics discipline has recently appeared[27-29], which allows a study of living objects on the basis of equilibrium thermodynamics and the physical chemistry of natural systems[28-31]. Macrothermodynamics is also based on the principles of macrokinetics, an alternative to the thermodynamics of systems close to equilibrium.

Modern hierarchical thermodynamic theory of the biological evolution and aging of living beings has developed upon thermodynamic models of quasi-closed quasi-equilibrium systems due to J.W. Gibbs' works (1873-1878)[32]. The methodologies of Gibbs' thermodynamics and the law of temporal hierarchies, or Gladyshev's law,[33-35] have been approximately extended to all hierarchies of biological systems, which are generally open ones[27,35-37]. Since thermodynamics of nonequilibrium processes (equilibrium thermodynamics and kinetics) deals with the rate of increase in production of entropy, therefore, nonequilibrium thermodynamics provides quantitative characteristic of the second law of thermodynamics[34].

The variation of the chemical composition of living beings in the course of ontogenesis and phylogenesis is a consequence of change in the mean specific value of the Gibbs function of formation for supramolecular and intermolecular interactions operating during the formation of supramolecular structures of an organism's tissues (functioning of biological systems) tends to a minimum. The value of Gibbs function tends toward a minimum as a result of the system's tendency to seek the equilibrium state; i.e., the tendency of the system (within constant temperature and constant pressure) to evolve towards a stable potential energy well, as defined by a minimum of free energy (Helmholtz).

The principle of the substance stability and feedback is applicable to any biological hierarchies. This principle explains the accumulation of a substance with chemically high energy capacity by biological systems in the course of evolution and aging of living beings. This energetic accumulation of substance forces water out of these systems. The thermodynamic theory of biological evolution and aging of living organisms provides general concepts pertaining to nutrition. These concepts will encourage and stimulate behavioral and dietary changes thermodynamically-favored towards the development of long and healthy human lives. Anti-aging diets should balance the composition and caloric value.

\subsection{Law of Temporal Hierarchy}

The law of temporal hierarchies substantiates the possibility of identifying, or discerning, quasi-closed monohierarchical systems or subsystems within open polyhierarchical biological systems[27,32,36-38]. As a rule, at the phenomenological level such investigations are carried out in terms of kinetic, or dynamical, linear thermodynamics. The law of temporal hierarchies can be presented as a series of strong inequalities. The direction of this series is towards increasing average life-spans of structures on going from lower to higher structures. The law of temporal hierarchies makes it possible to identify subsystems and to study their development (ontogenesis) and evolution (phylogenesis) by measuring the change (per unit of volume or mass) in the specific value of the Gibbs function of formation of the given higher hierarchical structure from structures of a lower level.

\subsection{Principle of Substance Stability}

The principle substance stability describes the tendency of temporal and structural natural hierarchy systems to seek out local equilibria (Van't Hoff) at all levels of the organization. These tendencies derived from the second law thermodynamics (the Clausius-Gibbs variation) in coordination with the Le Chatelier-Braun principle[32,36-41]. The principle of substance stability is determined by the limited energetic potential, i.e. the Gibbs potential energy, of associated interacting elementary structures at time scales relative to our capabilities.

The principle applied to molecular and supramolecular structures was named the principle of the stability of a chemical substance. This principle is also known as the principle of stability of matter, the principle of substance stability, the feedback principle, or Gladyshev's principle. Similar phenomena occur in molecular chromatographic columns; specifically, hydrophobic cells and columns[32, 39-41]. These columns accumulate substance with a high energy capacity.

For instant, the tumor cells have a lower ability for aggregation and they easily move in the body, which leads to the appearance of metastases. The tumor cell membrane is, apparently, formed from supramolecular structures of increased stability. Hence, the supramolecular stability of cell aggregates formed with the participation of tumor cells should be lowered according to the principle of substance stability. In order to increase the adhesive ability of the cells, the structure of membranes should be "diluted" and made less thermodynamically stable. This explains why experimental anticancer diets recommend the use of plant oils, fats of animals from cold seas, and other products containing residues of unsaturated low-melting-point fatty acids. The anti-tumor effect and the influence of some chemical substances on the supramolecular structures of nucleic acids can also be explained on the basis of such principle[32,37].

Socially, the relationship between the principle of substance stability and the elemental structures of any inside 
social hierarchy are defined as an understructure hierarchy; e.g., a hierarchy of organisms, groups of organisms[32]. For instant, the stronger the family ties and mutual understanding (understructure hierarchy) between family members, the less time they spend outside the family, i.e. the "overstructure hierarchy". It can be seen that hierarchical thermodynamics may apply appropriately to understand the human life as well.

Accordingly, the principle of substance stability corresponds with the rules of maintenance of stability of parties, unions, states, communities and nations[32,35]. Thus one can realize the age-old social management methods as "divide and rule". The history of mankind can be possibly predicted on the basis of the principles of hierarchical thermodynamics. In such case the quantitative thermodynamics of social hierarchy and the concept of sociological potential can be used[27].

Regarding connections between the principle of substance stability and politics, it is clear why people who are distinguished by their independence and audacity seek achieving power. These individuals use techniques and methods developed only by their own and unavailable, due to moral considerations, to the average cultured person. Nevertheless, having achieved a high position, these members of society begin, under favorable conditions, to come into conflict with similar members of society. Achieving great power requires not only a combination of favorable factors but also a person's intelligence, which, however, usually shows itself in various weird and particular activities.

Technologically, the rapid development of human race is associated with a better selection of energy-consuming systems and devices. These systems and devices, while making life easier for the people who own them and for society as a whole, invariably result to increase humanities' thermodynamic or sociological potential. However, in accordance with the principle of substance stability, humankind together with its technogenic environment as a single system will become with time unstable. This will lead to the partial degradation of the system and to its complete destruction. The laws of thermodynamics are relentless and they effect everywhere in universe extended to all hierarchies of matter $[32,35,36]$. Based on ecological theory, the earth is a self-regulating organism that adjusts to changes in order to maintain suitable conditions for life. Thus feedback between all hierarchical levels of biological world based on hierarchical thermodynamics should exist $[32,37,38]$.

\subsection{Supramolecular Thermodynamics and Anti-Aging Quality of Foodstuff}

From the thermodynamics laws, the supramolecular structures of a foodstuff (a substance) and the value of gerontological (anti-aging) quality of foodstuff depend on the Gibbs specific function or Gibbs specific free energy of the supramolecular or intermolecular formation of the condensed phase. This depends also on the change of specific enthalpy and entropy during the solidification of natural fat (oil), melting point and on the standard temperature[32]. The value of standard temperature must be lower than value of melting temperature. When the gerontological value of a food is evaluated, the choice of standard temperature is determined by the melting point of the lowest melting-point substance in the series of compared products. It is assumed that the low melting-point substances take part in the formation of corresponding low melting-point supramolecular structures in an organism's tissues. Such a correlation is used as an indicator of the anti-aging (gerontological) value of the food[32,38].

\subsection{Artificial Life and in Silico Simulation}

Today, the theoretical biology with the advances in mathematical physics, in modeling and in computer technologies permit an exploration of life "in silico". Computer simulations are used to explore 'Artificial Life' or 'A-Life' seeking life as it was on earth in a larger conceptual context of any possible form of life[42,43]. Work in A-Life emphasizes on the processes of living things rather than the material constituents of their structures[44]. However such studies emphasize on the organizational relationship between components rather than the components themselves. Focusing on the emerging age of "proteomics" - in the post human genome era- the complex functional interactions of the large array of cellular proteins are being studied[45]. In spite A-Life allows life to be subject of physical and chemical laws, it is too soon yet to know how important the contribution of the A-Life programs will be.

\subsection{Mathematical-Physics Modeling of Evolution}

The mathematical modeling of evolution was elaborated in several directions: life origin models, mathematical population genetics, models of evolution of genetic regulatory systems, as well as artificial life evolutionary models. These models give generalized descriptions of biological experiments. Some evolutionary models (life origin models, artificial life evolutionary models) provide us with more abstract pictures - they describe artificial evolutionary processes, not as we know them, but as they could be. Nevertheless, there are serious problems to be analyzed such as the problems of evolution of cybernetic, and computer-like "intelligent" features of biological organisms. The theoretical investigations of these problems could constitute the subject of "evolutionary biocybernetics". In general evolutionary modeling fields are:

Models of molecular-genetic systems origin which describe mathematically some hypothetical evolutionary stages of prebiological self-reproducing macromolecular systems.

Artificial life evolutionary models which analyze the evolution of artificial organisms.

Computer algorithms are used to solve practical problems of evolutionary methods of optimization.

General models of evolution describing some informational and cybernetic aspects of evolution. 
The Artificial Life models are incorporating in activities such as learning, neural networks and adaptive behavior.

\section{Conclusions}

Understanding of the physical-chemical basis of living systems has increased enormously in the past century giving a plausible definition of life. Living organisms are autopoietic systems: self-constructing, self-maintaining, energy-transducing autocatalytic entities. Information needed to construct the next generation of organisms is stabilized in nucleic acids that replicate within the context of whole cells and work with other developmental resources during the life-cycles of organisms. They are also systems capable of evolving by variation and natural selection: self-reproducing entities, whose forms and functions are adapted to their environment and reflect the composition and history of an ecosystem[14]. Such a perspective represents a fulfillment of the basic dual insights of Schrödinger near mid-century. Much remains to be elucidated about the relationships among the complex molecular systems of living entities, how they are constrained by the system as a whole as well as by physical laws and their action with evolutionary theory. Recently there is a sense of the importance of putting Schrödinger's program into a 'systems' context[46-58]. Work in A-Life seeking evidence of extra-terrestrial life may help the formulation of a more universal concept of life. The principle of the stability of chemical substances of the supramolecular structures of tissues makes it possible to understand the causes of the evolution of the biological world[3]. The emergence of life characteristics are complexity, metabolism, teleonomy, abundance and diversity, and especially their farfrom-equilibrium state, all derive directly from the kinetic character of the nucleic acid replicating reaction of complex living systems. From replicative chemistry point of view, efficient molecular replicators might derive from alternative carbon based systems, or even non-carbon ones. No attempt has been made to discuss the particular evolutionary stages from simple replicating molecules to complex biostructures. Thermodynamics governs the behavior of chemical processes, where life is consistent with the second law, both in living and non-living systems. According to the thermodynamic theory and experimental data, the specific value of the Gibbs function of the formation of supramolecular structures of organisms during ontogenesis and phylogenesis tends to a minimum. Living systems exhibit an extreme expression of kinetic control. So our kinetic perspective on life seems to lead to some interesting conclusion. Unified theories or even postulates are still needed to integrate strongly the molecular biology and biochemistry (microstructure) into the thermal physics (macrostructure) providing a better gain to understand the amazing phenomena: genesis and evolution of biological organization up to the human conscience.

\section{ACKNOWLEDGMENTS}

The author is grateful to Mrs. Safia El-Diasty for her fruitful discussion and to Dr. Nabil El-Faramawy and Dr. Nashwa Abdel Baki for their revision of the article.

\section{REFERENCES}

[1] S. J. Goerner, "Chaos and the Evolving Ecological Universe," Gordon \& Breach (1994)

[2] P. A. Corning, S. J. Kline, Syst. Res. Behav. Sci. Vol. 14 (1997) 1

[3] G. P. Gladyshev, Entropy 1 (1999) 9

[4] S. J. Gould, Sci. Amer. 271 (1994) 62

[5] R. A. Fisher, The Genetical Theory of Natural Selection, 2nd edition, Dover Publications, New York, 1958

[6] A. I. Oparin, "The Origin of Life," (1936) Translation of the 1929 Russian publication by S. Morgulis. New York: Macmillan

[7] J. B. S. Haldane, "The origin of life," Rationalist Animal (1929). Reprinted as an appendix in J. D. Bernal 1967, The Origin of Life. Cleveland: World

[8] R. Dawkins, "The Selfish Gene," Oxford: Oxford University Press (1989)

[9] W. Gilbert, Nature 319 (1986) 618

[10] H. J. Morowitz, "The Emergence of Everything: How the World Became Complex," New York: Oxford University Press (2002)

[11] B. H. Weber, "Emergence of life and biological selection from the perspective of complex systems dynamics." In G. van de Vijver, S.N. Salthe, and M. Delpos (eds.) Evolutionary Systems: Biological and Epistemological Perspectives on Selection and Self-Organization. Dordrecht: Kluwer (1998)

[12] R. J. P. Williams, J. J. R. Frasto da Silva, Biochem. Biophys. Res. Commun. 297 (2002) 689

[13] R. J. P. Williams, J. J. R. Frasto da Silva, J. Theo. Bio., 220 (2003) 323

[14] F. M. Harold, "The Way of the Cell: Molecules, Organisms and the Order of Life," New York: Oxford University Press (2001)

[15] A. I. Oparin, "The Origin of Life on Earth," 3rd Edn. Edinburgh: Oliver \& Boyd (1957)

[16] M. Eigen, Naturwissenschaften, 58 (1971) 465

[17] M. Eigen, "Steps Toward Life: a Perspective on Evolution," Oxford: Oxford University Press (1992)

[18] S. Lifson, J. Mol. Evol. 44 (1997) 1

[19] A. Process, J. Theo. Biol. 220 (2003) 393 
[20] F. G. Hopkins, "The dynamic side of biochemistry," Report of the British Association 1913: 652-658, reprinted in Nature 92: 213-223, and in Needham and Baldwin 1949, pp. 136-159

[21] E. Schrödinger, "What is Life? The Physical Aspect of the Living Cell," Cambridge: Cambridge University Press (1944)

[22] A. J. Lotka, " Proceedings of the National Academy of Sciences Vol. 8 (1922) 147

[23] J. D. Watson, F. H. Crick, Nature 171 (1953) 964

[24] I. Prigogine, G. Nicolis and A. Babloyants, Phys. Today 25 (1972) 25

[25] I. Prigogine, Impact of Science on Society, Vol. XXIII (1973) 169

[26] K. G. Denbigh, Brit. J. Phil. Sci. 40 (1989) 323

[27] G. P. Gladyshev, J. Theo. Biol. 75 (1978) 425

[28] G. P. Gladyshev, Zh. Fiz. Khim. 61 (1987) 2289

[29] G. P. Gladyshev, J. Biol. Systems 1 (1993) 115

[30] M. Calvin, "Chemical Evolution," Clarendon Press: Oxford, 1969

[31] Ch. Tanford, The Hydrophobic Effect and the Organization of Living Matter. In: "Origin of Life. The Central Concepts," Deamer, D.W.; Fleischaker, G.R., Eds.; Jones and Bartlett Publishers Inc.: Boston-London, 1994

[32] G. P. Gladyshev, N. N. Semenov, Int. J. Appl. Math. Stat. 11 (2007) 52

[33] J. W. Gibbs, "The Collected Works of J. Willard Gibbs. Thermodynamics," New York: Longmans, Green and Co., Vol. 1 (1928) 55

[34] D. N. Zubarev, Papers on thermodynamics. In: Physical Encyclopedia. Moscow: Soviet Encyclopedia, 1990, Vol. 1-5

[35] G. P. Gladyshev, The second law of thermodynamics and the evolution of living systems. Journal of Institute of Human Thermodynamics (The report in: ECOS, 18-th International Conference on Efficiency, Cost, Optimization, Simulation and Environmental Impact of Energy Systems, NTNU, Trondheim, Norway, June 20-23, 2005), Vol. 1, Issue 7, p. 68-81

[36] G. P. Gladyshev, Thermodynamic Theory of the Evolution of Living Beings. N.Y., Nova Sci. Publ. Inc. 1997

[37] G. P. Gladyshev, Int. J. Modern Phys. B 18 (2004) 801

[38] G. P. Gladyshev, Prog. Reaction Kinet. Mechan. 28 (2003) 157

[39] G. P. Gladyshev, Electron. J. Math. Phys. Sci. 2 (2002) 1
[40] G. P. Gladyshev, Entropy 1 (1999) 55

[41] G. P. Gladyshev, Bio. Bull. 5 (1998) 533

[42] C. G. Langton, “Artificial Life," Redwood City CA: Addison-Wesley (1989)

[43] C. G. Langton, “Artificial Life: An Overview," Cambridge MA: MIT Press (1995)

[44] C. Emmeche, "The Garden in the Machine: The Emerging Science of Artificial Life," Princeton: Princeton University Press (1994)

[45] A. Kumar, M. Snyder, Nature 415 (2002) 12

[46] R. Rosen, "Life Itself: A Comprehensive inquiry into the Nature, Origin, and Fabrication of Life," New York: Columbia University Press (1991)

[47] R. Rosen, "Essays on Life Itself," New York: Columbia University Press (2000)

[48] S. A. Kauffman, "The Origins of Order: Self-Organization and Selection in Evolution," New York: Oxford University Press (1993)

[49] S. A. Kauffman, "At Home in the Universe: The Search for the Laws of Self-Organization and Complexity," New York: Oxford University Press (1995)

[50] S. A. Kauffman, "Investigations," New York: Oxford University Press (2000)

[51] D. J. Depew, B. H. Weber, "Darwinism Evolving: System Dynamics and the Genealogy of Natural Selection," Cambridge MA: MIT Press (1995)

[52] B. H. Weber, D. J. Depew, Bio. Philos. 11(1996) 33

[53] B. H. Weber, D. J. Depew, "Developmental systems, Darwinian evolution, and the unity of science." In S. Oyama, P.E. Griffiths, and R.D. Gray Cycles of Contingency: Developmental Systems and Evolution, Cambridge MA: MIT Press (2001)

[54] R. E. Ulanowicz, "Ecology, The Ascendent Perspective," New York: Columbia University Press (1997)

[55] R. J. P. Williams, J. J. R. Frasto da Silva, "Bringing Chemistry to Life: From Matter to Man," Oxford: Oxford University Press (1999)

[56] R. J. P. Williams, J. J. R. Frasto da Silva, Biochem. Biophys. Res. Commun. 297 (2002) 689

[57] R. J. P. Williams, J. J. R. Frasto da Silva, J. Theo. Bio., 220 (2003) 323

[58] H. J. Morowitz, "The Emergence of Everything: How the World Became Complex," New York: Oxford University Press (2002) 